\title{
Efficacy of zoledronic acid in treatment of teoarthritis is dependent on the disease progression stage in rat medial meniscal tear model
}

\author{
De-gang $\mathrm{YU}^{1, \text { \# }}$, Bo $\mathrm{YU}^{2, \#}$, Yuan-qing MAO ${ }^{1, \#}$, Xin ZHAO ${ }^{1}$, Xiao-qing WANG ${ }^{1}$, Hui-feng DING ${ }^{1}$, Lei CAO ${ }^{1}$, Guang-wang LIU $^{1}$, \\ Shao-bo NIE ${ }^{3}$, Shen $\mathrm{LIU}^{4}$, Zhen-an ZHU ${ }^{1, *}$ \\ ${ }^{1}$ Department of Orthopedics, Shanghai Ninth People's Hospital, Shanghai Jiao Tong University, School of Medicine, Shanghai 200011 , \\ China; ${ }^{2}$ Department of Orthopedics, Affiliated Hospital of Shandong University of Traditional Chinese Medicine, Ji-nan 250014, China; \\ ${ }^{3}$ Department of Orthopedics, PLA General Hospital, Beijing 100853, China; ${ }^{4}$ Department of Orthopedics, Shanghai Sixth People's Hos- \\ pital, Shanghai Jiao Tong University, Shanghai 200233, China
}

Aim: To investigate whether the stage of osteoarthritis $(\mathrm{OA})$ progression influenced the efficacy of the third-generation bisphosphonate zoledronic acid in a rat medial meniscal tear model.

Methods: Medial meniscal tear (MMT) was surgically induced in adult male Sprague Dawley rats. Zoledronic acid (ZOL, 100 $\mu \mathrm{g} / \mathrm{kg}$, sc, twice a week) was administered starting immediately, early (from 4 weeks) or late (from 8 weeks) after OA induction. The degeneration of articular cartilage was evaluated with toluidine blue $\mathrm{O}$ staining. Subchondral bone remodeling was evaluated with $\mathrm{X}$-ray micro-CT scanning. Joint pain was measured with respect to weight-bearing asymmetry. Calcitonin gene-related peptide (CGRP) expression in dorsal root ganglia (DRGs) was examined using immunofluorescence analysis. The afferent neurons in DRGs innervating the joint were identified by retrograde labeling with fluorogold.

Results: Progressive cartilage loss was observed during 12 weeks after OA induction. Subchondral bone remodeling manifested as increased bone resorption at early stage (4 weeks), but as increased bone accretion at advanced stages ( 8 weeks). Immediately and early ZOL administration significantly improved subchondral microstructural parameters, attenuated cartilage degeneration, reduced weight-bearing asymmetry and CGRP expression, whereas the late ZOL administration had no significant effects.

Conclusion: The stage of OA progression influences the efficacy of ZOL in treating joint degeneration and pain. To obtain the maximum efficacy, bisphosphonate treatment should be initiated in rat with early stages of OA pathogenesis.

Keywords: osteoarthritis; zoledronic acid; bisphosphonate; pain; subchondral bone; rat medial meniscal tear model

Acta Pharmacologica Sinica (2012) 33: 924-934; doi: 10.1038/aps.2012.28; published online 21 May 2012

\section{Introduction}

Osteoarthritis (OA), a leading cause of disability in the elderly $^{[1]}$, is characterized by a progressive loss of articular cartilage and abnormalities in subchondral bone and surrounding soft tissue adaptations ${ }^{[2]}$, with chronic pain being the main clinical symptom ${ }^{[3]}$. The search for effective agents that attenuate joint degeneration and relieve joint pain has been challenging.

For decades, articular cartilage has been the focus of major basic research and pharmaceutical therapies, although alterations in the subchondral bone were generally considered to

\footnotetext{
"These authors contributed equally to this paper.

* To whom correspondence should be addressed.

E-mail zhuzhenan2006@126.com

Received 2012-02-12 Accepted 2012-03-05
}

occur secondary to cartilage degeneration ${ }^{[4,5]}$. However, it is increasingly accepted that subchondral bone plays an important role in the pathogenesis of OA. Accumulating evidence from clinical and animal studies has demonstrated that during OA progression, subchondral bone turnover increases dramatically, with increased bone resorption occurring early, and increased bone accretion occurring later in the disease $\mathrm{e}^{[6-8]}$. Meanwhile, the biochemical composition and biomechanical properties of the subchondral bone are altered ${ }^{[9,10]}$. Therefore, because of an intimate biological and mechanical relationship with articular cartilage, the presence of abnormal subchondral bone influences the integrity of the overlying cartilage ${ }^{[11-13]}$. In addition, abnormalities in subchondral bone, such as exposure of the subchondral bone plate, bone attrition, and bone marrow lesions, have been associated with knee pain ${ }^{[14-17]}$.

Consequently, targeting subchondral bone with bone-mod- 
ifying agents would be expected to retard joint degeneration and alleviate pain. Bisphosphonates, the most commonly used agents for osteoporosis treatment, are a promising therapy for OA because of their ability to inhibit osteoclastic bone absorption $^{[18]}$. However, the ability of bisphosphonates to preserve the structural integrity of subchondral bone and attenuate cartilage degeneration that has been seen in animal studies has not been consistently confirmed by clinical data ${ }^{[19-22]}$, which is in part attributed to deficiencies in evaluation criteria or experimental design. Subchondral bone properties are dynamic with disease progression. Therefore, patients with different stages of subchondral bone alterations could have been included in the studies and may have contributed to the inconsistent clinical findings. We hypothesized that the stage of disease progression, especially the stage of subchondral bone remodeling, could influence the efficacy of bisphosphonate therapy.

Many animal models have been developed to experimentally reproduce OA. The most commonly used rat medial meniscal tear (MMT) model demonstrates dynamic subchondral bone changes. Therefore, the cartilage- and bonepreserving activities of therapeutic agents can be evaluated in this model ${ }^{[23]}$. Moreover, this model manifests changes in pain behavior, neuron phenotype and neuropeptide expression $^{[24-26]}$, which, taken together, represent a model of the joint pain associated with OA. Therefore, in the present study, we used the established rat MMT model and initiated zoledronic acid (ZOL, a third-generation bisphosphonate) treatment at different stages after OA induction to evaluate the efficacy of ZOL treatment on joint degeneration and pain. Pain behavior, cartilage histology, subchondral microarchitecture, and the expression of calcitonin gene-related peptide (CGRP), a painrelated neuropeptide, in the dorsal root ganglia (DRG) were evaluated.

\section{Materials and methods}

Animals

Adult male Sprague Dawley rats (Sino-British Sippr/BK Lab

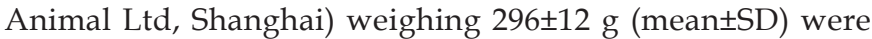
used for these experiments. Animals were group-housed on a 12-h light/dark cycle with food and water ad libitum. All experimental procedures were approved by the Animal Care and Experiment Committee of Shanghai Jiaotong University School of Medicine and were consistent with the National Institutes of Health Guide for the Care and Use of Laboratory Animals. Every effort was made to minimize the number of animals used and their suffering.

\section{Induction of $\mathrm{OA}$}

After acclimatization for 1 week, rats were anesthetized with $10 \%$ chloral hydrate in phosphate buffered saline (PBS, $0.01 \mathrm{~mol} / \mathrm{L})$. The MMT model was induced as previously described $^{[27]}$. Briefly, the medial collateral ligament of the right knee was transected, and the medial meniscus was reflected proximally toward the femur and cut through at its narrowest point. For the controls (Sham), the wounds were closed after exposing the medial collateral ligament.

\section{Experimental design}

A total of 154 animals were used for the present study. In the preliminary experiment, of the total number of animals used, 64 animals were used to observe the changes in cartilage and subchondral bone during OA progression. The animals were sacrificed at 2, 4, 8, and 12 weeks after OA induction $(n=8$ at each time point). Another 40 animals were used to confirm the positive effects of ZOL in the MMT model and to choose a dose of ZOL that better preserved subchondral bone. ZOL (Aclasta $^{\circledR}$, Novartis Pharma Stein AG) was administered subcutaneously twice a week at low dose $10 \mu \mathrm{g} / \mathrm{kg}$ body weight (MMT/ZolL) and high dose $100 \mu \mathrm{g} / \mathrm{kg}$ body weight (MMT/ $\mathrm{ZolH})$. The same volume of normal saline was given to the Sham animals (Sham/NS) and MMT control animals (MMT/ NS). The animals ( $n=10$ per group) were sacrificed at 4 weeks after $\mathrm{OA}$ induction.

Based on the above results, ZOL treatment was initiated at three stages following OA induction: immediately (ZOL0), early (4 weeks, ZOL4) and late (8 weeks, ZOL8). ZOL was administered subcutaneously twice a week at $100 \mu \mathrm{g} / \mathrm{kg}$ body weight. The same volume of normal saline was given to the Sham animals (Sham/NS) and MMT control animals (MMT/ NS). All five groups of animals ( $n=10$ per group) were sacrificed at 12 weeks following the induction of OA. The experimental design is shown as Scheme 1.

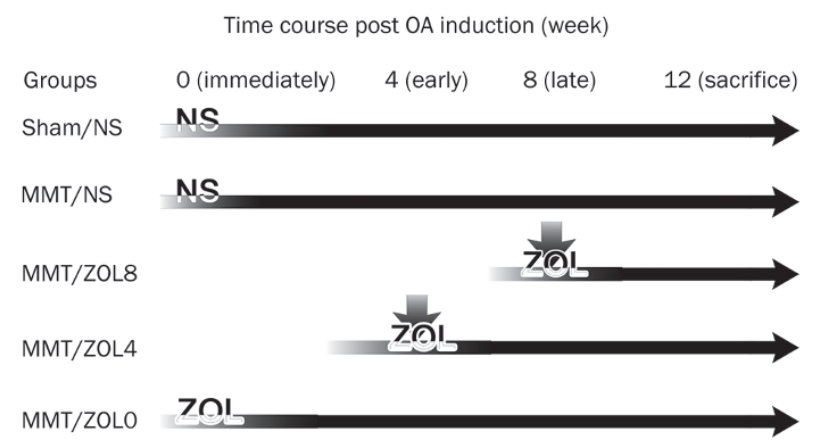

Scheme 1. ZOL treatment initiated at three stages following $O A$ induction.

\section{Pain behavior testing}

Pain behavior was measured as weight-bearing asymmetry between the OA-induced (right) and contralateral (left) limb using an incapacitance meter (IITC Life Sciences, Woodland Hills, CA, USA) as previously described ${ }^{[24]}$. Briefly, rats were placed in an angulated Perspex container so that each hind paw rested on a separate transducer pad. The force exerted by each hind limb was averaged over a 5-s period. Each data point is the mean of three 5-s readings. Changes in weightbearing asymmetry were evaluated one day before and once a week after OA induction. The percentage of weight placed on the right hind limb was determined using the formula below $^{[28]}$. 
$\%$ of weight on right limb=

$$
\frac{\text { weight on right limb }}{\text { weight on right limb+weight on left limb }} \times 100
$$

\section{Retrograde neuronal labeling}

Afferent neurons in DRGs innervating the joint were identified by retrograde labeling with fluorogold (FG). To this end, $5 \mu \mathrm{L}$ of $2 \%$ FG (Sigma, 39286) in $0.1 \mathrm{~mol} / \mathrm{L}$ PBS was injected intraarticularly into the right knee of an anesthetized rat using a 33-gauge needle and a Hamilton microsyringe one week prior to the end of the experiment ${ }^{[25]}$.

\section{Tissue preparation}

After anesthesia, rats were transcardially perfused with 250 $\mathrm{mL}$ of heparinized saline $(10 \mathrm{IU} / \mathrm{mL})$ followed by $300 \mathrm{~mL}$ of ice-cold, fresh $4 \%$ paraformaldehyde (PFA) in $0.1 \mathrm{~mol} / \mathrm{L}$ PBS. Lumbar L3, L4, and L5 DRGs were harvested and fixed in $4 \%$ PFA for $3 \mathrm{~h}$ and then cryoprotected in $30 \%$ sucrose in $0.1 \mathrm{~mol} / \mathrm{L}$ PBS overnight at $4{ }^{\circ} \mathrm{C}$. DRGs were then sliced into $12-\mu \mathrm{m}$ sections with every sixth section collected on the same glass slide and stored at $-80^{\circ} \mathrm{C}$. The entire knee joint was also dissected for histological and micro-CT imaging.

\section{Micro-CT imaging and analysis}

After fixation in $4 \%$ PFA for $48 \mathrm{~h}$, the knee joints were scanned by X-ray microcomputed tomography (micro-CT) $(\mu \mathrm{CT} 80$; SCANCO Medical AG) with an isotropic voxel resolution of $10 \mu \mathrm{m}$. A portion $(2.0 \mathrm{~mm}$ ventrodorsal length) of the loadbearing region at the medial tibial plateau was identified as a region of interest. The following three-dimensional structural parameters were calculated: bone volume fraction (BV/TV), trabecular thickness (Tb.Th), trabecular spacing (Tb.Sp), trabecular number (Tb.N), and connectivity density (CD).

\section{Histological analysis}

After being imaged by micro-CT, the knee joints were decalcified in 10\% EDTA for 3 weeks. The joints were bisected along the collateral ligament in the frontal plane, and both sections were embedded in the same paraffin block. The samples were cut into 5- $\mu \mathrm{m}$ sections. On the basis of the OARSI recommendations for the histological assessment of osteoarthritis in the rat ${ }^{[23]}$, three sections from each knee at 200-mm steps were stained with toluidine blue $\mathrm{O}$ and subsequently evaluated for cartilage degeneration. Three sections at $200-\mathrm{mm}$ steps were chosen for H\&E staining and for evaluation of the synovial reaction. For toluidine blue $\mathrm{O}$ staining, the sections were stained with $0.04 \% w / v$ toluidine blue O (Sigma, T0394) in 0.1 $\mathrm{mol} / \mathrm{L}$ sodium acetate $(\mathrm{pH} 4.0)$ for $10 \mathrm{~min}$, rinsed briefly, airdried and mounted.

\section{Immunofluorescence analysis}

Slides containing every sixth section of L3, L4, and L5 DRGs were chosen for immunofluorescence analysis for CGRP and subsequent Nissl staining to quantify the total number of neurons in each section. The sections were first pre-incubated in $0.1 \mathrm{~mol} / \mathrm{L}$ PBS containing 1\% Triton X-100 and 10\% normal goat serum (NPBST) for $30 \mathrm{~min}$ at room temperature (RT) and then incubated with rabbit polyclonal CGRP (1:2000, Sigma, C8198) overnight at $4{ }^{\circ} \mathrm{C}$ in NPBST. After washing three times with NPBST, the sections were incubated with antirabbit-Alexa 488 (1:500, Molecular Probes, A11034) for $1 \mathrm{~h}$ at RT. After rinsing 3 times with PBS, the sections were labeled with fluorescent Nissl stain (1:50, Molecular Probes, N-21482). Finally, the sections were mounted with Fluoromount$\mathrm{G}^{\mathrm{TM}}$ (SBA, 0100-01) and visualized. All of the images were acquired using a fluorescence microscope (Leica DM 4000B) with BioQuant OSTEO II software (BioQuant Image Analysis Corporation, Nashville, TN). An entire image of every DRG section was acquired at $200 \times$ magnification with automatic sequential imaging. The immunofluorescent images were analyzed with Image-ProPlus 6.0 (Media Cybernetics). The total numbers of FG-labeled neurons, the percentage of neurons immunolabeled for CGRP, and the total number of Nissllabeled neurons were determined. The results were averaged for each individual rat.

\section{Statistical analysis}

All behavioral testing, micro-CT statistical analysis, cartilage scoring and neuron counting was performed by blinded authors who were unaware of the treatments given. The results are presented as the mean \pm standard deviation (SD). A comparison between groups was performed with one-way analysis of variance using the LSD post-hoc test. $P$ values less than 0.05 were considered statistically significant.

\section{Results}

Generally, cartilage changes occurred and worsened in a timedependent manner between 2 and 12 weeks post $\mathrm{OA}$ induction (Figure 1). At 2 weeks, matrix and chondrocyte loss affected the superficial and upper middle zone, and at 4 weeks, these changes deepened into the deep zone and extended into more than $1 / 3$ zone of the medial tibial plateau. Severe changes occurred at 8 weeks with full thickness degeneration into the tidemark, which become more widespread at 12 weeks. Subchondral bone structure of the medial tibial plateau showed dynamic alterations following OA induction from increased bone resorption at early stages ( 2 and 4 weeks) to increased bone accretion at advanced stages ( 8 and 12 weeks) (Figure 2). Compared with the Sham groups, BV/TV was significantly decreased at 2 and 4 weeks and increased at 8 and 12 weeks post-operation. These changes were accompanied by $\mathrm{Tb}$.Th changing from thinner ( 2 and 4 weeks) to thicker (8 and 12 weeks), Tb.Sp from broader ( 2 and 4 weeks) to more narrow (8 and 12 weeks), and $\mathrm{Tb} . \mathrm{N}$ and $\mathrm{CD}$ being reduced at all times.

Compared with MMT/NS animals, cartilage matrix and chondrocyte loss were partly reversed in animals with ZOL administration at both doses, and additional efficacy was observed in the MMT/ZOLH group (Figure 3). Abnormal microarchitecture parameters, such as the noticeably reduced BV/TV, Tb.N, Tb.Th, CD, and increased Tb.Sp seen in the MMT/NS group, were well preserved by high-dose ZOL treatment and partly preserved with low-dose therapy. In 
A
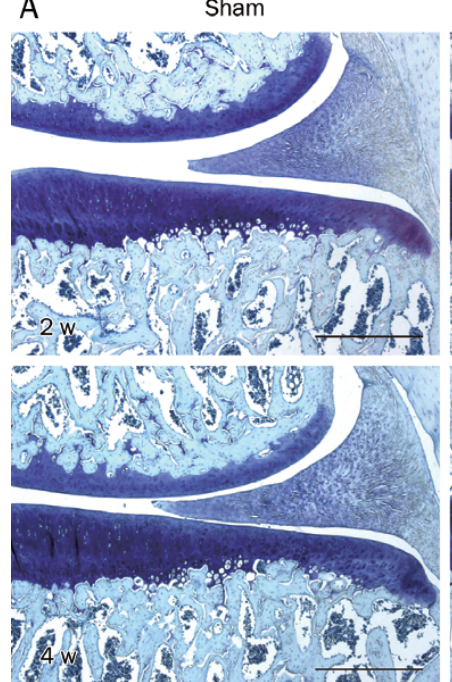

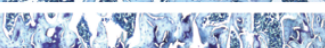

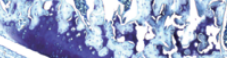

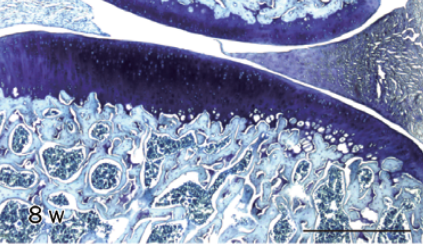

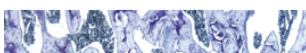
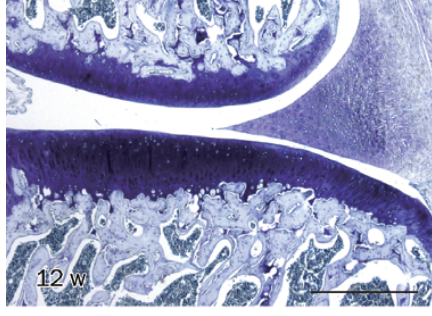

B

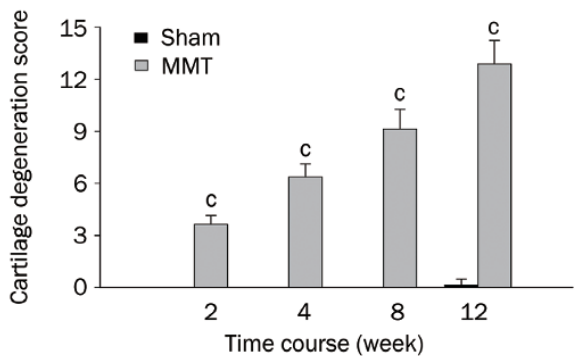

Figure 1. Changes in articular cartilage during OA progression. (A) Representative images with toluidine blue 0 staining. Bar=400 $\mu \mathrm{m}$. (B) Cartilage degeneration score. The comparison between groups was performed with an independent-samples $t$-test. Mean \pm SD. $n=8 .{ }^{c} P<0.01$ vs Sham group.

addition, marked osteosclerosis was observed at the tibial metaphysis in animals treated with ZOL, which was induced by ZOL inhibiting osteoclasts in the growth plate (Figure 4).

\section{Pain behavior}

As shown in Figure 5, there was a marked reduction in hind limb weight-bearing 1 week post-OA induction, which was almost completely resolved by 2 weeks in all of the groups. Subsequently, Sham/NS animals returned to normal weightbearing, whereas MMT animals (MMT/NS, MMT/ZOL0, MMT/ZOL4, MMT/ZOL8) experienced weight-bearing asymmetry that gradually became worse in a time-dependent manner. Compared with MMT/NS controls at any time point tested, rats that received early ZOL therapy showed less weight-bearing asymmetry (MMT/ZOL0 and MMT/ ZOL4) with an increased benefit observed in rats that received therapy immediately after OA induction. However, delayed administration of ZOL (MMT/ZOL8) did not yield a statistically significant difference. Specifically, 12 weeks post-OA induction, the MMT/ZOL0, MMT/ZOL4 and MMT/ZOL8 animals placed $45.21 \% \pm 1.07 \%(P<0.01), 43.66 \% \pm 1.39 \%(P<0.05)$ and $42.15 \% \pm 1.25 \%(P>0.05)$, respectively, of their hind limb weight on the right leg compared with $41.97 \% \pm 0.93 \%$ in the MMT/NS animals.

\section{Articular cartilage degeneration}

As indicated by toluidine blue $\mathrm{O}$ staining and cartilage degeneration scoring (Figure 6), widespread and profound cartilage matrix and chondrocyte loss were observed in MMT/NS animals at 12 weeks; however, this loss was significantly reduced by ZOL administration in MMT/ZOL0 animals and moderately reduced in MMT/ZOL4 animals. No statistically significant improvement was observed in the MMT/ZOL8 animals. Specifically, the cartilage degeneration score was $7.25 \pm 1.28$ $(P<0.01), 10.13 \pm 1.55(P<0.01)$, and $12.0 \pm 1.69(P>0.05)$ for the MMT/ZOL0, MMT/ZOL4, and MMT/ZOL8 animals, respectively, compared with $12.13 \pm 1.55$ for the MMT/NS animals.

\section{Synovial reaction}

The synovial reaction was evaluated based on the OARSIrecommended scoring system. As shown in Figure 7S and $8 \mathrm{~S}$, the synovial reaction was similar among all the groups. The synovial lining comprised only 1-2 layers of cells, and no inflammatory cell infiltration was observed.

\section{Subchondral bone remodeling}

Representative images of knee joints obtained from micro-CT scanning are shown in Figure 7B. Generally, MMT resulted in increased bone accretion and cyst formation at 12 weeks, whereas ZOL treatment reversed these changes in a stagedependent manner. The bone cysts observed in the MMT/NS animals were absent in the MMT/ZOL0 and MMT/ZOL4 animals and appeared to be smaller in the MMT/ZOL8 animals, although the size of the cysts was not quantified. In addition, marked osteosclerosis was present at the tibial metaphysis in animals treated with ZOL, which occurred as a result of ZOL treatment inhibiting osteoclasts in active growth plates. The analysis of subchondral bone microstructure indexes is shown in Figure 7C. Compared with the Sham/NS animals, the subchondral bone of the medial tibial plateau in MMT/ NS animals was significantly altered. The alterations included increased $\mathrm{BV} / \mathrm{TV}$, thickened $\mathrm{Tb}$.Th, narrowed $\mathrm{Tb} . \mathrm{Sp}$, and 
A
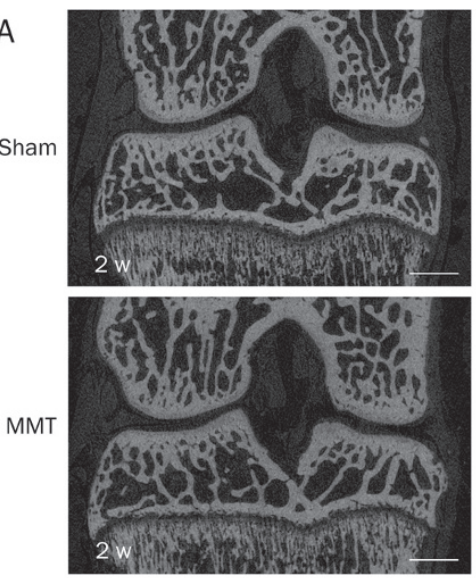
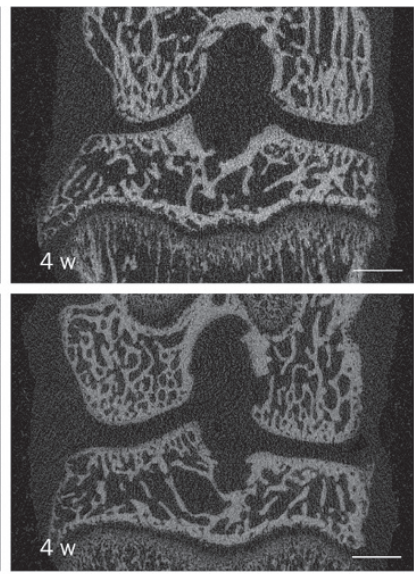
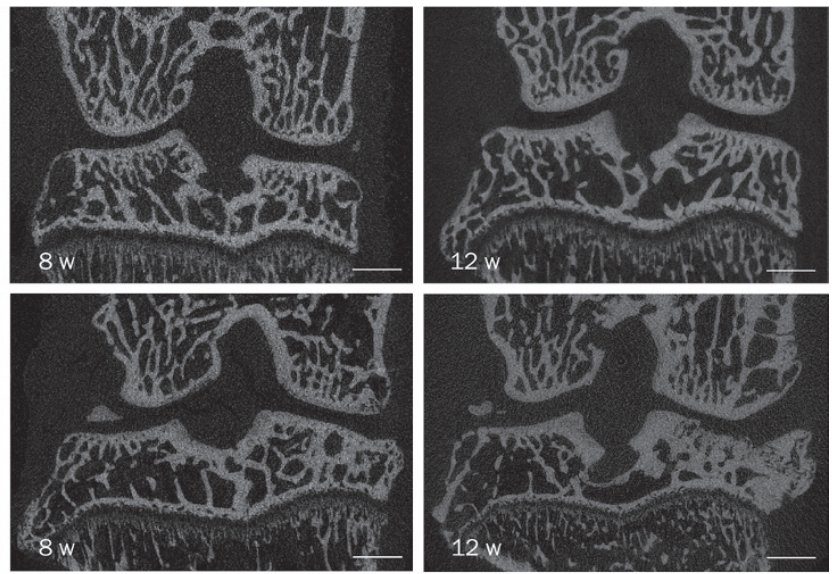

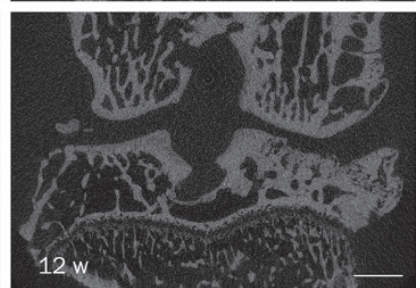

B
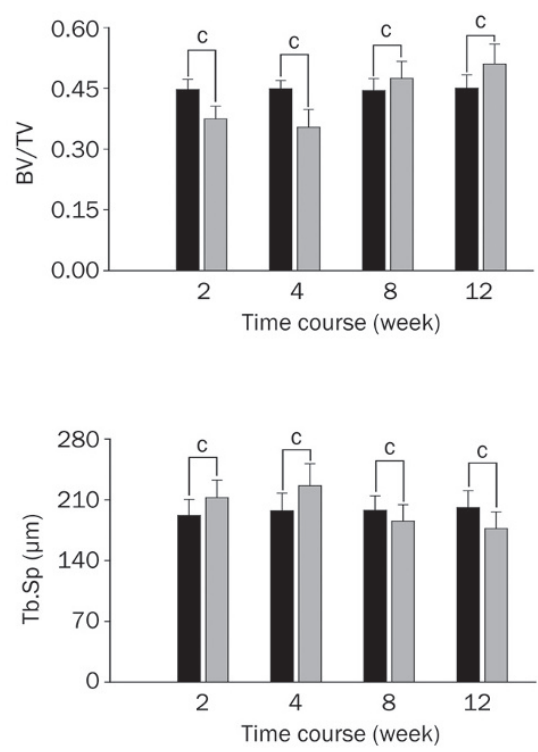
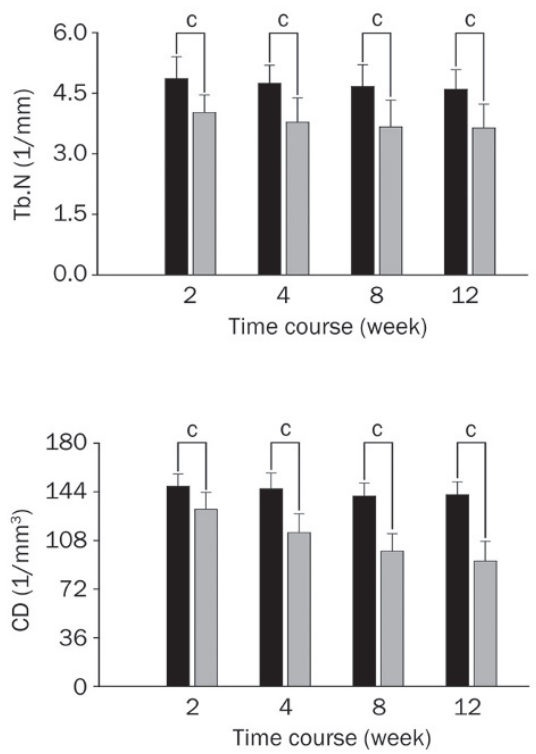

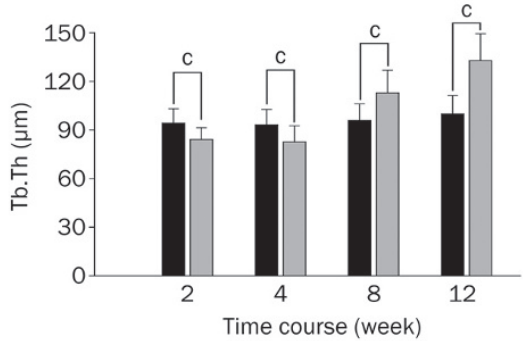

- Sham

$\square$ MMT

Figure 2. Changes in subchondral bone during OA progression. (A) Representative images of the knee obtained with micro-CT scanning, Bar=1000 $\mu \mathrm{m}$. (B) Analysis of subchondral bone microstructure parameters. The comparison between groups was performed with an independent-samples $t$-test. Mean \pm SD. $n=8 .{ }^{\circ} P<0.01$ vs Sham group.

reduced Tb.N and $C D$, which were significantly preserved by immediate ZOL administration (MMT/ZOL0), moderately preserved by early administration (MMT/ZOL4), and not altered by late administration (MMT/ZOL8).

\section{CGRP expression in DRG neurons}

As shown in Figure 8, the total neuron number, as determined by fluorescent Nissl staining, was similar among all five groups $(P>0.05)$. Retrograde labeling through intra-articular injection of FG allowed the tracing of afferent neurons that innervate the knee joint in L3, L4, and L5 DRGs. The total number of FG-labeled neurons was not significantly different among the groups $(P>0.05)$. Among the FG-labeled neurons, the percentage of CGRP-positive neurons in MMT/NS rats $(48.9 \% \pm 3 \%)$ was increased compared with that observed in the Sham/NS rats $(40.7 \% \pm 3 \%, P<0.01)$. This ratio was significantly reduced by immediate (MMT/ZOL0, $42.8 \% \pm 2.8 \%$,
$P<0.01$ ) and early (MMT/ZOL4, 44.6\% $\pm 2.9 \%, P<0.01) \mathrm{ZOL}$ administration but not by administration at advanced stages despite a decreasing trend (MMT/ZOL8, $47.5 \% \pm 2.4 \%, P>0.05$ ).

\section{Discussion}

The present study demonstrated that cartilage degeneration and subchondral bone remodeling in the rat MMT model occurred in a time-dependent manner. With disease progression, subchondral bone remodeling shifted from increased bone resorption at an early stage to bone accretion at an advanced stage. These results were consistent with those of earlier published studies ${ }^{[8,19]}$. Similar to previous studies ${ }^{[19,29]}$, the current study showed that bisphosphonates demonstrated a protective effect on cartilage and bone changes related to OA in a dose-dependent manner. To better elucidate the timedependent effects of the therapy, the higher dose was chosen. We found that immediate ZOL administration significantly, 

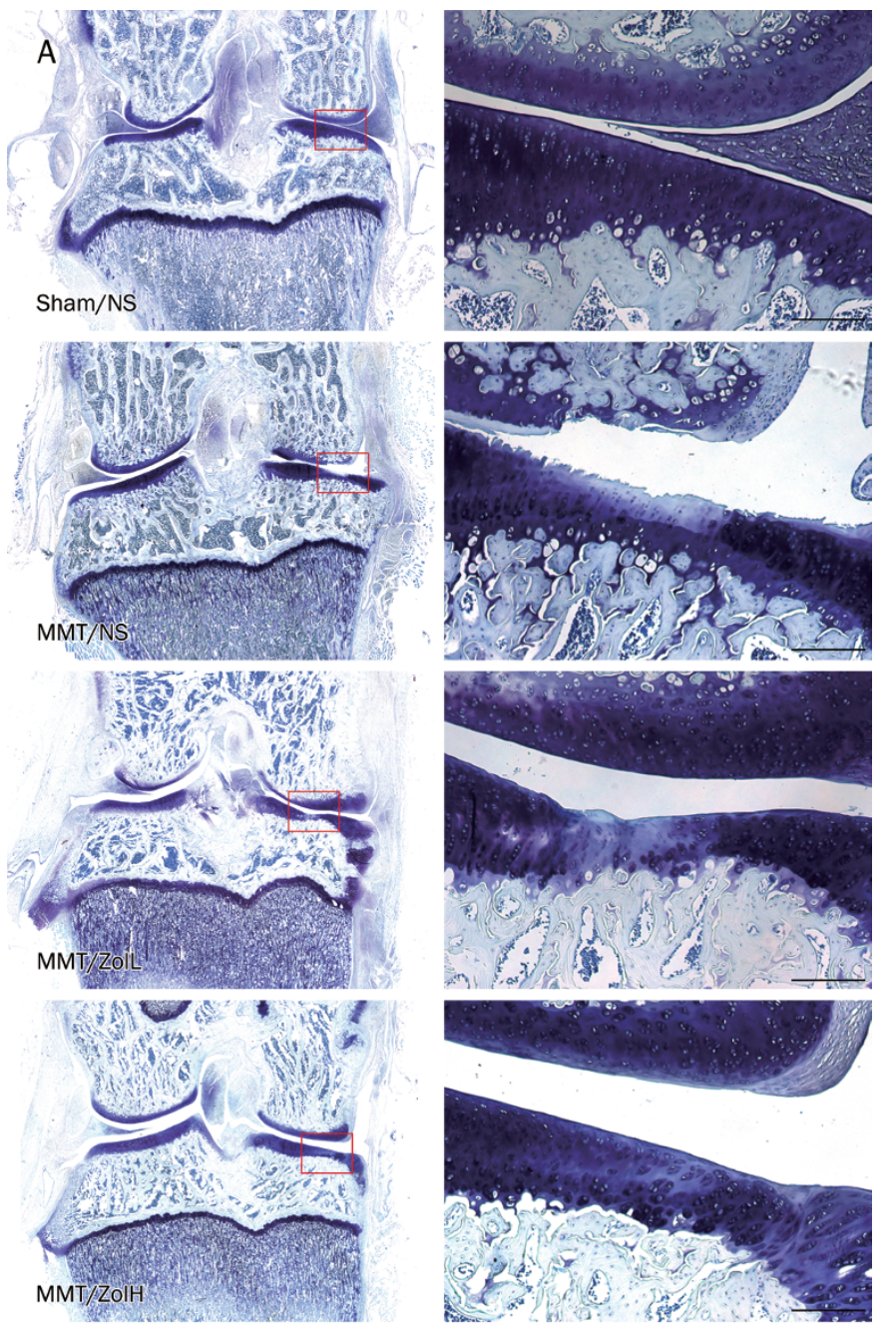

ship of articular cartilage and subchondral bone ${ }^{[10,13,22]}$, the abnormal remodeling of subchondral bone would aggravate the degeneration of overlying articular cartilage; thus, retarded bone remodeling would slow the degeneration of overlying articular cartilage ${ }^{[9,11,12]}$. However, because subchondral bone properties are dynamic during disease progression, the abnormal subchondral bone cannot always be modified. Our data demonstrated that subchondral bone absorption is increased at an early stage of OA. If ZOL treatment is initiated at this stage, abnormalities in subchondral bone can be reversed to a large extent and then cartilage degeneration would be retarded accordingly. Conversely, at an advanced stage, subchondral bone accretion was increased mainly due to excessive mechanical stress ${ }^{[30-32]}$. If ZOL therapy is given at this stage, subchondral bone remodeling would not be reversible, and articular cartilage degeneration would continue.

To better identify the effects of ZOL on joint pain, pain behavior was measured as weight-bearing asymmetry between operated and contralateral hind limbs, and the expression of CGRP in DRG neurons innervating the joint was measured. CGRP is a marker of sensory neurons expressed by $\mathrm{C}$ and A $\delta$ primary afferent fibers and is mainly involved

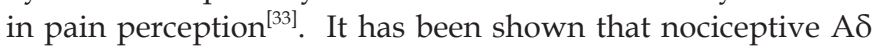
and $C$ fibers innervate the capsule, ligaments, periosteum and subchondral bone of the joint ${ }^{[3]}$. Osteoarthritic conditions activate the nociceptive system, and CGRP is elevated in the $\mathrm{DRGs}^{[25,34]}$. A decrease in the number of CGRP-positive DRG neurons was also consistent with improved pain behavior ${ }^{[25,35]}$. In accordance with a previous study, our data show that the percentage of CGRP-positive neurons was markedly increased at advanced stages in the MMT model, whereas ZOL treatment initiated at early stages reduced the expression of CGRP. These findings, combined with the results of pain behavior studies, indicate that ZOL treatment initiated at an early stage could relieve joint pain.

Currently, the origin of pain in osteoarthritis is poorly understood. Synovitis has been found to be associated with knee pain severity ${ }^{[14,17]}$. However, consistent with a previous study ${ }^{[24]}$, histological analysis of the MMT joint in the present study did not reveal any synovial hyperplasia (Figures 75 and 85 ). Hence, the weight-bearing asymmetry and increased CGRP expression after MMT surgery indicate that OA structural changes, rather than synovitis, cause joint pain. ZOL treatment initiated early, but not late, retards cartilage degeneration and subchondral bone remodeling, leading us to believe that ZOL reduces joint pain by inhibiting OA structural changes. Adult articular cartilage is normally an avascular tissue $^{[3]}$; therefore, abnormal subchondral bone may be the main source of algogenesis. This hypothesis is supported by findings that knee pain is correlated with abnormalities in subchondral bone $\mathrm{e}^{[14-17]}$ and by the following findings. Osteochondral vascular density increased with increasing cartilage deterioration severity and clinical disease activity scores ${ }^{[36]}$. Sensory nerves were present within vascular channels at the osteochondral junction in $\mathrm{OA}^{[37]}$. Therefore subchondral nerves may be exposed to painful stimuli in OA, which would and early to some degree, improved subchondral microstructural parameters, decreased the cartilage degeneration score, and reduced weight-bearing asymmetry and CGRP expression. Late administration demonstrated no statistically significant efficacy.

Because of the intimate biological and mechanical relation- 
A

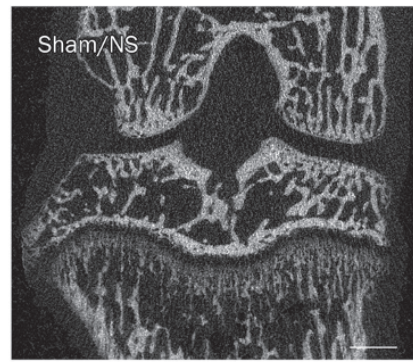

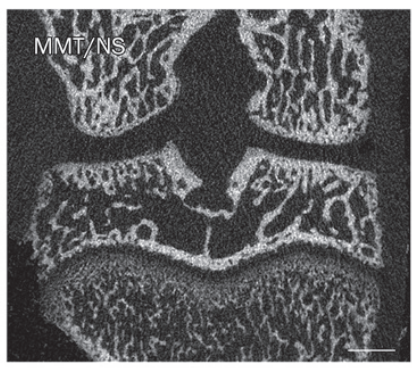
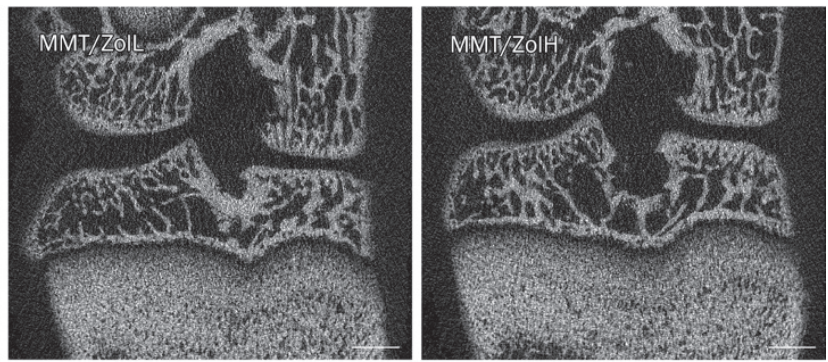

B
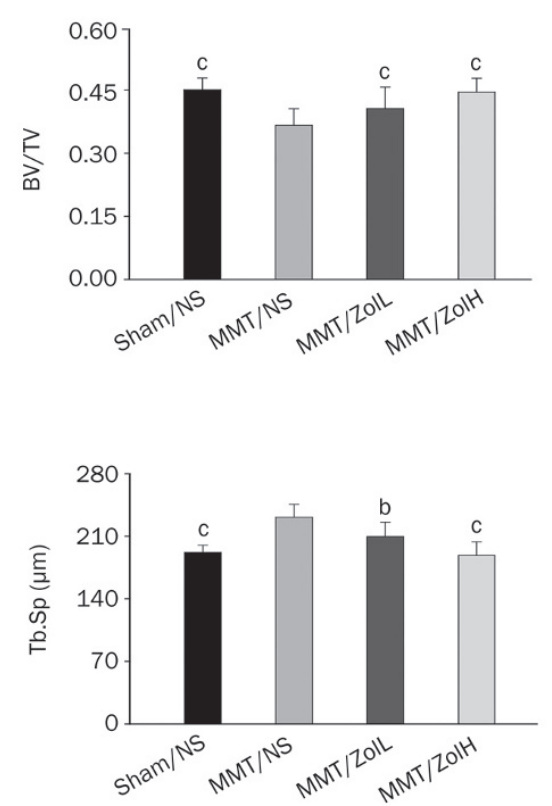
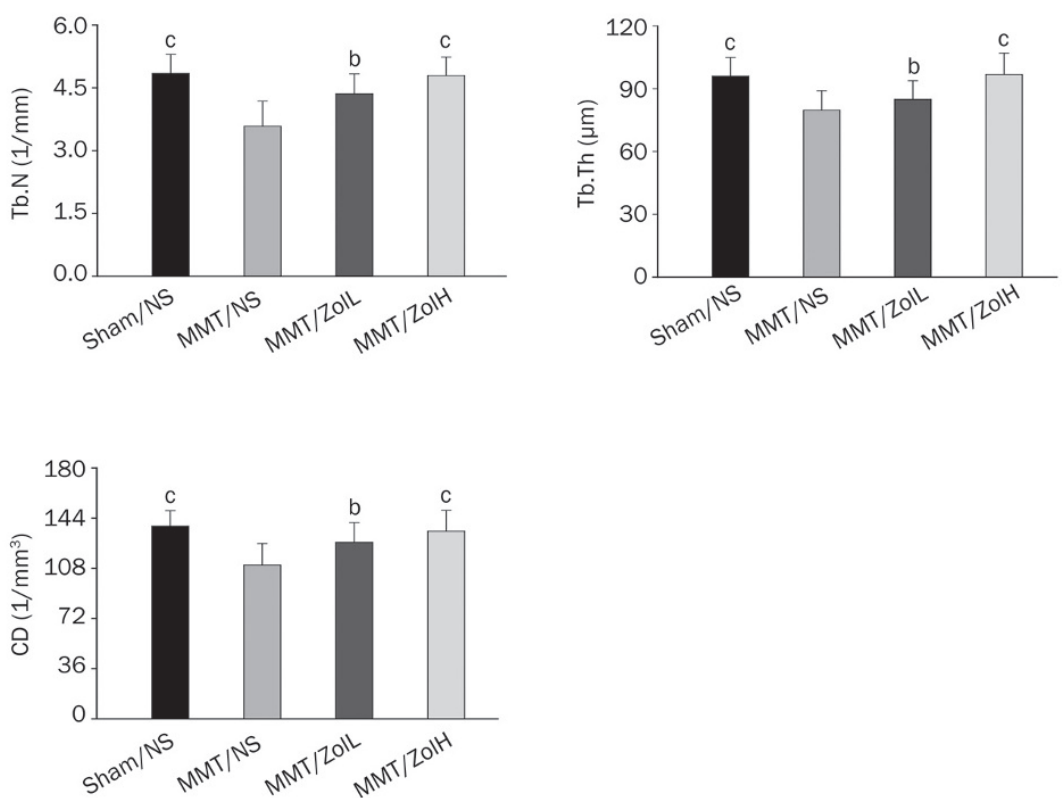

Figure 4. The effects of low dose $10 \mu \mathrm{g} / \mathrm{kg}$ body weight (MMT/ZolL) and high dose $100 \mu \mathrm{g} / \mathrm{kg}$ body weight (MMT/ZolH) on subchondral bone remodeling. (A) Representative images of the knee obtained using micro-CT scanning, Bar=1000 $\mu \mathrm{m}$. (B) Analysis of subchondral bone microstructure parameters. The comparison between groups was performed with one-way analysis of variance using the Tukey post-hoc test. Mean \pm SD. $n=10$. ${ }^{\mathrm{b}} P<0.05,{ }^{\mathrm{c}} P<0.01$ vs MMT/NS group.

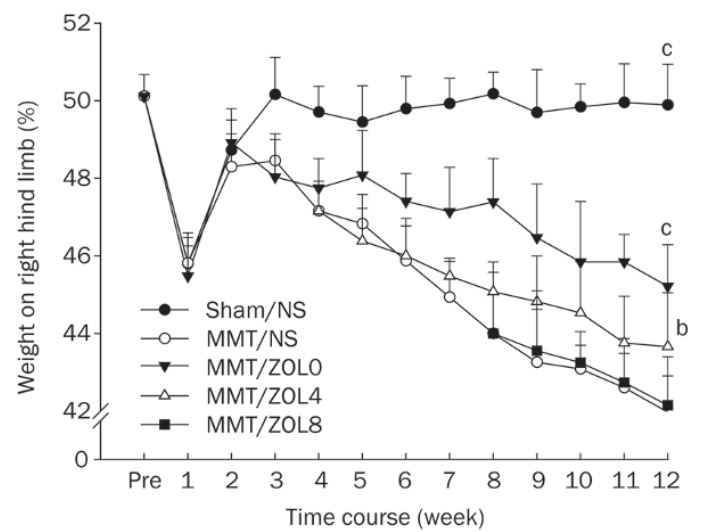

Figure 5. The effect of ZOL $100 \mu \mathrm{g} / \mathrm{kg}$ treatment initiated at different stages on pain behavior, as measured by the percentage of weight borne on the right hind limb. $n=10$ per group. ${ }^{b} P<0.05,{ }^{c} P<0.01$ vs MMT/NS group. lead to weight-bearing pain, the most common symptom in OA patients ${ }^{[3]}$. Immediate pain relief is obtained by total or unicompartmental knee arthroplasty ${ }^{[38]}$, in which most of the affected subchondral plate is excised. Therefore, we hypothesize that the potential mechanism by which bisphosphonates reduce joint pain mainly lies in improving cartilage and subchondral bone integrity and accordingly inhibiting subchondral neurovascular invasion. However, other potential mechanisms may be involved. For example, the acidic environment created by osteoclasts could cause bone pain during subchondral bone remodeling ${ }^{[19,39]}$. Nevertheless, weight-bearing asymmetry and CGRP expression were similar in MMT/NS rats and MMT/ZOL8 rats. However, subtle differences may be detected with a more sophisticated behavior apparatus, such as a computerized incapacitance meter system and gait analysis system, to measure neuron electrophysiology and detect additional neuropeptides in DRGs and the spinal cord.

In the present study, ZOL was phase-administered based 


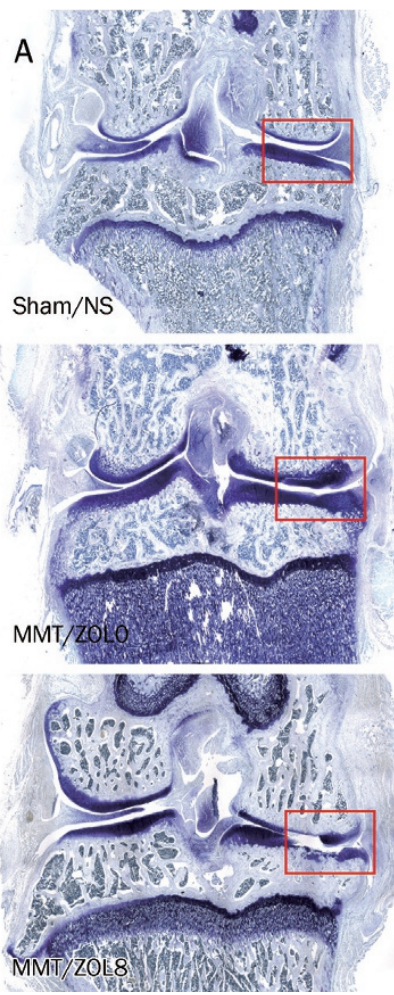

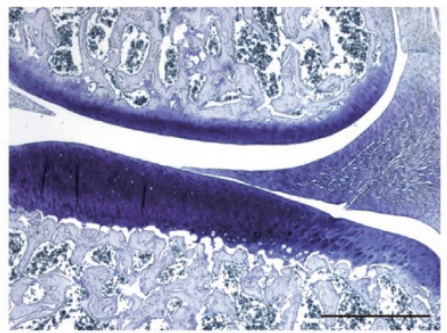
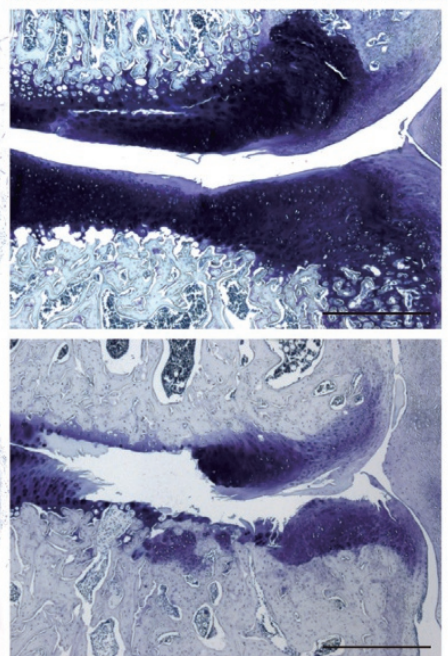
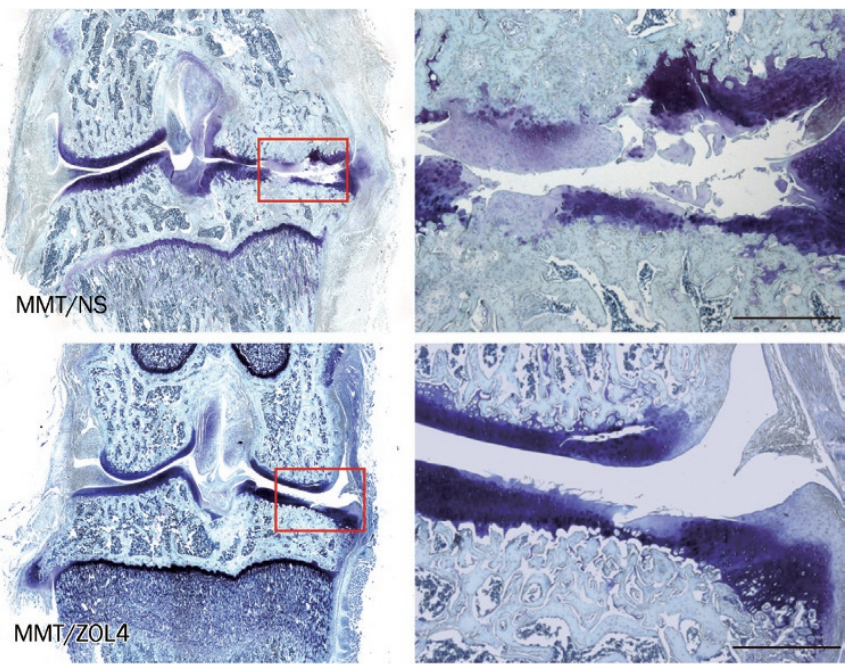

B

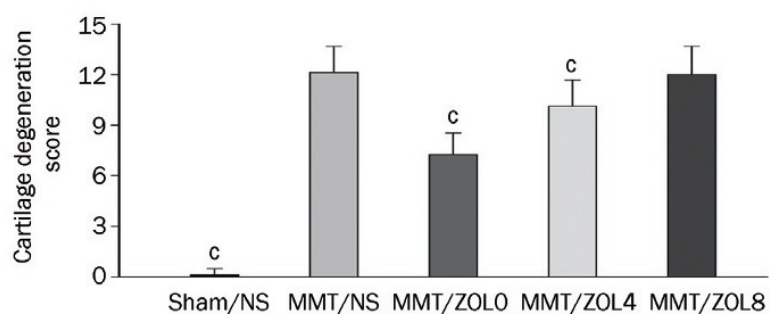

Figure 6. The effect of ZOL $100 \mu \mathrm{g} / \mathrm{kg}$ treatment initiated at different stages on cartilage degeneration. (A) Representative images of toluidine blue 0 staining. Bar $=400 \mu \mathrm{m}$. (B) Cartilage degeneration score. $n=10$ per group. ${ }^{\mathrm{c}} P<0.01 \mathrm{vs}$ MMT/NS group.

on changes in the subchondral bone microarchitecture indices from micro-CT scanning. If combined with changes in bone formation and resorption biomarkers during OA progression, a better understanding of the relationship between the efficacy of ZOL treatment and the stage of disease progression, especially in the situation of subchondral bone remodeling, could be obtained.

In conclusion, our study demonstrated, using an established rat MMT OA model, that the stage of OA progression influences the efficacy of ZOL therapy on joint degeneration and pain. ZOL treatment initiated immediately, and to some degree early, significantly inhibited subchondral bone remodeling, attenuated cartilage degeneration, and alleviated joint pain; no statistically significant efficacy was observed if therapy was initiated at a later stage, when significant cartilage degeneration and subchondral bone accretion were already present. Similarly, to obtain the maximum efficacy, bisphosphonate treatment should be initiated in rat at an early stage of $\mathrm{OA}$, rather than in individuals with advanced stages of OA pathogenesis.

\section{Acknowledgements}

This work was supported by grants from the National Natural Science Foundation of China (№ 30700852 and 30973038), the Fund for Key Disciplines of Shanghai Municipal Education Commission (J50206) and the Program for the Shanghai Key Laboratory of Orthopedic Implants (Grant № 08DZ2230330). The majority of the research was completed in the Shanghai
Key Laboratory of Orthopedic Implants.

\section{Author contribution}

De-gang YU and Bo YU carried out most of the behavioral testing and immunohistochemical experiments. Yuan-qing $\mathrm{MAO}$ and Xin ZHAO performed micro-CT imaging experiments. Xiao-qing WANG, Hui-feng DING, Lei CAO, Guangwang LIU, Shao-bo NIE, Shen LIU, and Zhen-an ZHU conceived the study, designed the experiments, participated in data analysis and helped to draft the manuscript. Xiao-qing Wang and Guang-wang LIU performed H\&E staing and synovial reaction evaluation in the supplementary study.

\section{Supplementary Information}

Supplementary figures is available at the Acta Pharmacologica Sinica website.

\section{References}

1 Holman HR, Lorig KR. Overcoming barriers to successful aging. Selfmanagement of osteoarthritis. West J Med 1997; 167: 265-8.

2 Lane NE, Brandt K, Hawker G, Peeva E, Schreyer E, Tsuji W, et al. OARSI-FDA initiative: defining the disease state of osteoarthritis. Osteoarthritis Cartilage 2011; 19: 478-82.

3 Hunter DJ, McDougall JJ, Keefe FJ. The symptoms of osteoarthritis and the genesis of pain. Med Clin North Am 2009; 93: 83-100.

4 Felson DT, Neogi T. Osteoarthritis: is it a disease of cartilage or of bone? Arthritis Rheum 2004; 50: 341-4.

5 Mansell JP, Collins C, Bailey AJ. Bone, not cartilage, should be the major focus in osteoarthritis. Nat Clin Pract Rheumatol 2007; 3: 306-7. 
A

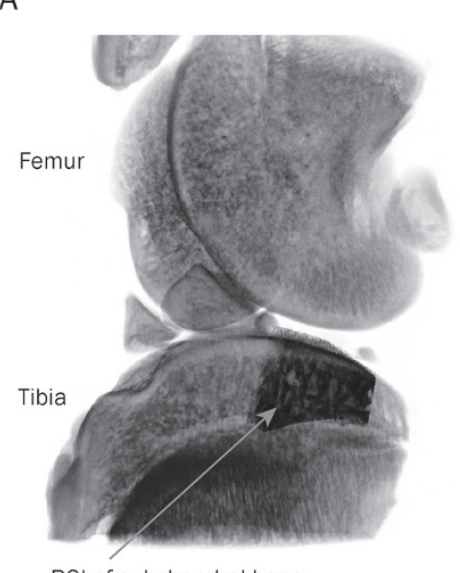

$\mathrm{ROI}$ of subchondral bone
$\mathrm{B}$
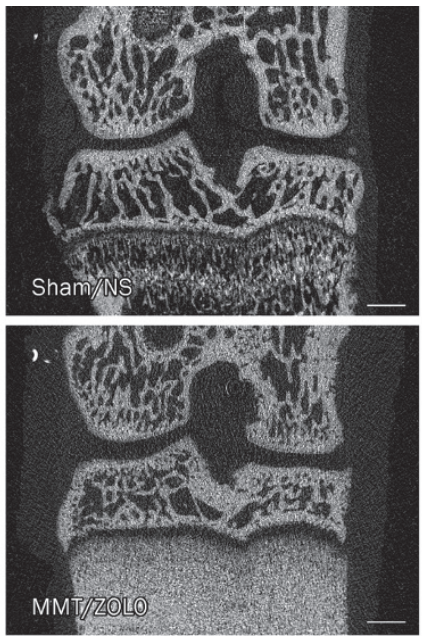
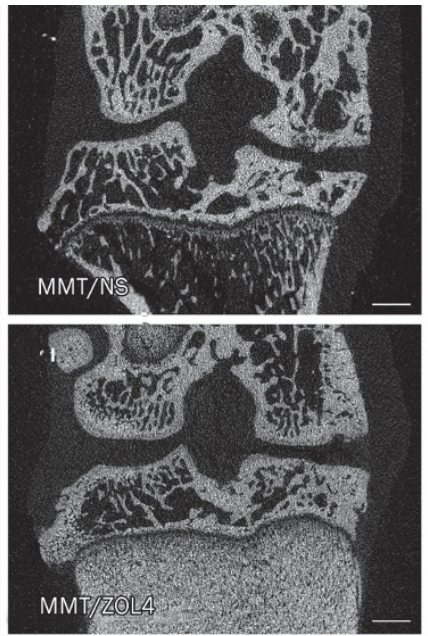

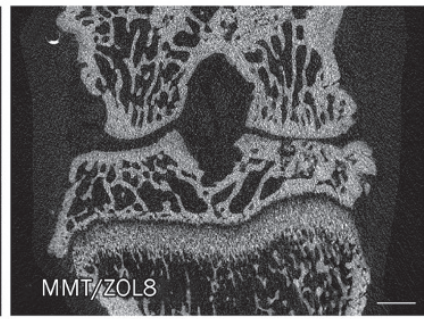

C
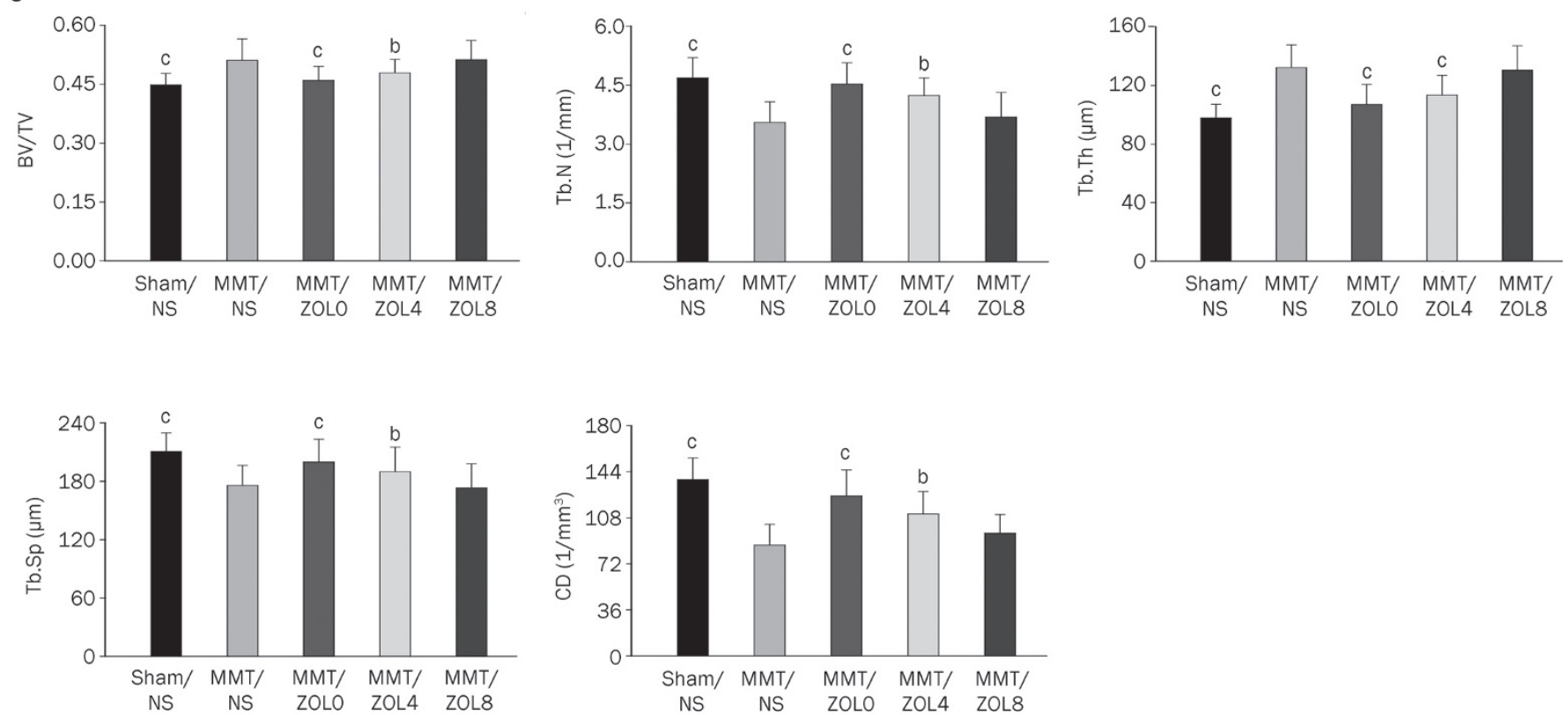

Figure 7. The effect of ZOL $100 \mu \mathrm{g} / \mathrm{kg}$ treatment initiated at different stages on subchondral bone remodeling. (A) The load-bearing region at the medial tibial plateau was taken as a region of interest (ROI). (B) Representative micro-CT images of the knee. Bar=1000 $\mu \mathrm{m}$. (C) Analysis of subchondral bone microstructure. $n=10$ per group. ${ }^{b} P<0.05,{ }^{c} P<0.01$ vs MMT/NS group.

6 Karsdal MA, Leeming DJ, Dam EB, Henriksen K, Alexandersen P, Pastoureau $\mathrm{P}$, et al. Should subchondral bone turnover be targeted when treating osteoarthritis? Osteoarthritis Cartilage 2008; 16: 638-46.

7 Goldring SR. Role of bone in osteoarthritis pathogenesis. Med Clin North Am 2009; 93: 25-35.

8 Kwan Tat S, Lajeunesse D, Pelletier JP, Martel-Pelletier J. Targeting subchondral bone for treating osteoarthritis: what is the evidence? Best Pract Res Clin Rheumatol 2010; 24: 51-70.

9 Bailey AJ, Mansell JP, Sims TJ, Banse X. Biochemical and mechanical properties of subchondral bone in osteoarthritis. Biorheology 2004; 41: 349-58.

10 Burr DB. Anatomy and physiology of the mineralized tissues: role in the pathogenesis of osteoarthrosis. Osteoarthritis Cartilage 2004; 12: S20-30.

11 Calvo E, Castaneda S, Largo R, Fernandez-Valle ME, Rodriguez-
Salvanes F, Herrero-Beaumont G. Osteoporosis increases the severity of cartilage damage in an experimental model of osteoarthritis in rabbits. Osteoarthritis Cartilage 2007; 15: 69-77.

12 Goldring MB, Goldring SR. Articular cartilage and subchondral bone in the pathogenesis of osteoarthritis. Ann N Y Acad Sci 2010; 1192: 230-7.

13 Lories RJ, Luyten FP. The bone-cartilage unit in osteoarthritis. Nat Rev Rheumatol 2011; 7: 43-9.

14 Felson DT. The sources of pain in knee osteoarthritis. Curr Opin Rheumatol 2005; 17: 624-8.

15 Neogi T, Felson D, Niu J, Nevitt M, Lewis CE, Aliabadi P, et al. Association between radiographic features of knee osteoarthritis and pain: results from two cohort studies. BMJ 2009; 339; b2844.

16 Moisio K, Eckstein F, Chmiel JS, Guermazi A, Prasad P, Almagor O, et al. Denuded subchondral bone and knee pain in persons with knee 

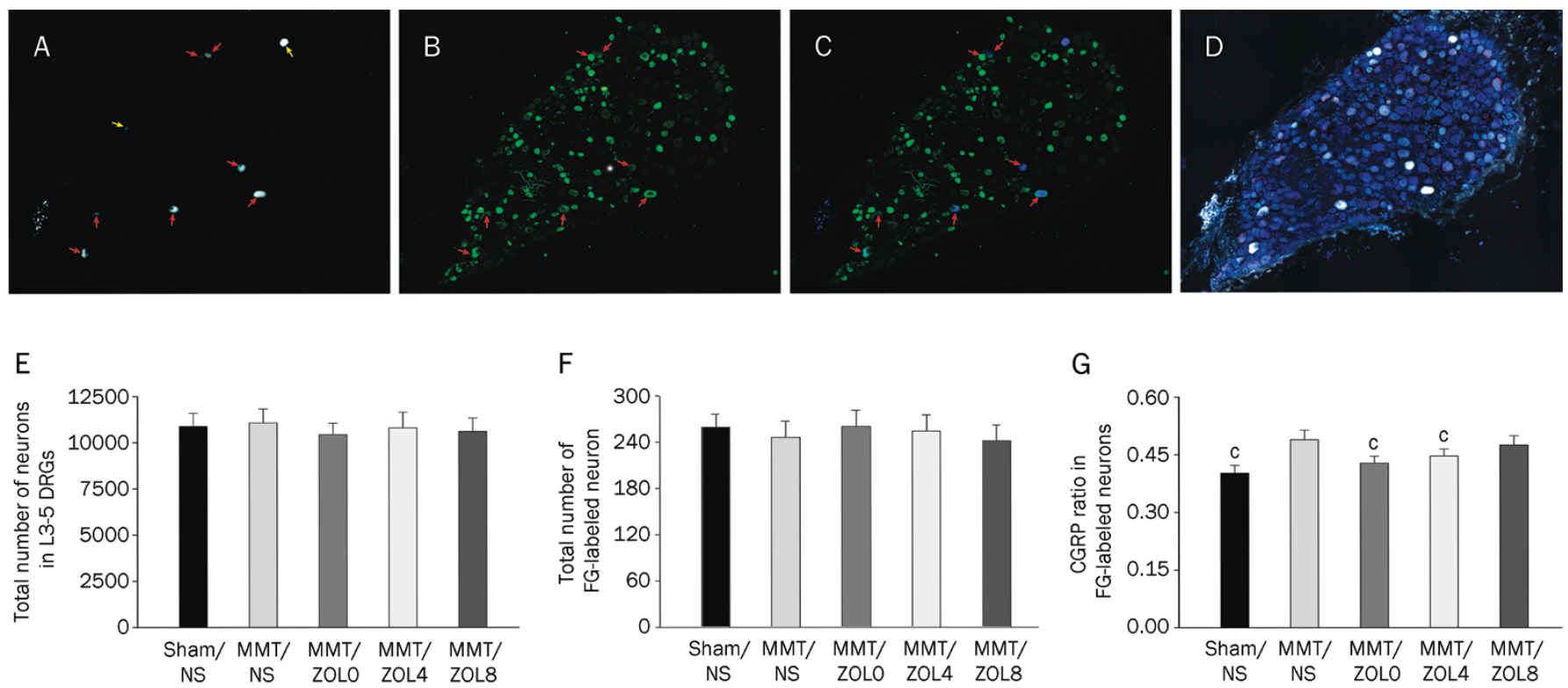

Figure 8. The effects of ZOL $100 \mu \mathrm{g} / \mathrm{kg}$ treatment on the expression of CGRP in DRGs. Representative images of the same DGR section retrograde labeled with FG (A), labeled with CGRP (B), labeled with FG and CGRP (C) and stained with fluorescent nissl (D). The red arrowheads indicate FG-labeled neurons that are CGRP-positive, the yellow arrowheads indicate CGRP-negative. The total number of neurons (E), the total number of FG-labeled neurons $(F)$, and the percentage of CGRP-positive neurons out of the total number of FG-labeled neurons, in L3, L4, and L5 DRGs (G). The results were averaged for each individual rat. $n=10$ per group. ${ }^{\circ} P<0.01$ vs MMT/NS group.

osteoarthritis. Arthritis Rheum 2009; $60: 3703-10$.

17 Wenham CY, Conaghan PG. Imaging the painful osteoarthritic knee joint: what have we learned? Nat Clin Pract Rheumatol 2009; 5: 149-58.

18 Lash RW, Nicholson JM, Velez L, Van Harrison R, McCort J. Diagnosis and management of osteoporosis. Prim Care 2009; 36: 181-98.

19 Hayami T, Pickarski M, Wesolowski GA, McLane J, Bone A, Destefano J, et al. The role of subchondral bone remodeling in osteoarthritis: reduction of cartilage degeneration and prevention of osteophyte formation by alendronate in the rat anterior cruciate ligament transection model. Arthritis Rheum 2004; 50: 1193-206.

20 Carbone LD, Nevitt MC, Wildy K, Barrow KD, Harris F, Felson D, et al. The relationship of antiresorptive drug use to structural findings and symptoms of knee osteoarthritis. Arthritis Rheum 2004; 50: 351625.

21 Saag KG. Bisphosphonates for osteoarthritis prevention: "Holy Grail” or not? Ann Rheum Dis 2008; 67: 1358-9.

22 Castaneda S, Roman-Blas JA, Largo R, Herrero-Beaumont G. Subchondral bone as a key target for osteoarthritis treatment. Biochem Pharmacol 2012; 83: 315-23.

23 Gerwin N, Bendele AM, Glasson S, Carlson CS. The OARSI histopathology initiative-recommendations for histological assessments of osteoarthritis in the rat. Osteoarthritis Cartilage 2010; 18: S24-34.

24 Bove SE, Laemont KD, Brooker RM, Osborn MN, Sanchez BM, Guzman RE, et al. Surgically induced osteoarthritis in the rat results in the development of both osteoarthritis-like joint pain and secondary hyperalgesia. Osteoarthritis Cartilage 2006; 14: 1041-8.

25 Ochiai N, Ohtori S, Sasho T, Nakagawa K, Takahashi K, Takahashi N, et al. Extracorporeal shock wave therapy improves motor dysfunction and pain originating from knee osteoarthritis in rats. Osteoarthritis Cartilage 2007; 15: 1093-6.

26 Im HJ, Kim JS, Li X, Kotwal N, Sumner DR, van Wijnen AJ, et al. Alteration of sensory neurons and spinal response to an experimental osteoarthritis pain model. Arthritis Rheum 2010; 62: 2995-3005.

27 Janusz MJ, Bendele AM, Brown KK, Taiwo YO, Hsieh L, Heitmeyer SA. Induction of osteoarthritis in the rat by surgical tear of the meniscus: Inhibition of joint damage by a matrix metalloproteinase inhibitor. Osteoarthritis Cartilage 2002; 10: 785-91.

28 Strassle BW, Mark L, Leventhal L, Piesla MJ, Jian Li X, Kennedy JD, et al. Inhibition of osteoclasts prevents cartilage loss and pain in a rat model of degenerative joint disease. Osteoarthritis Cartilage 2010; 18: 1319-28.

29 Buckland-Wright JC, Messent EA, Bingham CO, 3rd, Ward RJ, Tonkin C. A 2 yr longitudinal radiographic study examining the effect of a bisphosphonate (risedronate) upon subchondral bone loss in osteoarthritic knee patients. Rheumatology 2007; 46: 257-64.

30 Fukuda Y, Takai S, Yoshino N, Murase K, Tsutsumi S, Ikeuchi K, et al. Impact load transmission of the knee joint-influence of leg alignment and the role of meniscus and articular cartilage. Clin Biomech 2000; 15: 516-21.

31 Lindsey CT, Narasimhan A, Adolfo JM, Jin H, Steinbach LS, Link T, et al. Magnetic resonance evaluation of the interrelationship between articular cartilage and trabecular bone of the osteoarthritic knee. Osteoarthritis Cartilage 2004; 12: 86-96.

32 Cox LG, van Rietbergen B, van Donkelaar CC, Ito K. Bone structural changes in osteoarthritis as a result of mechanoregulated bone adaptation: a modeling approach. Osteoarthritis Cartilage 2011; 19: 676-82.

33 Hokfelt T. Neuropeptides in perspective: the last ten years. Neuron 1991; 7: 867-79.

34 Hunter DJ, McDougall JJ, Keefe FJ. The symptoms of osteoarthritis and the genesis of pain. Rheum Dis Clin North Am 2008; 34: 62343.

35 Staton PC, Wilson AW, Bountra C, Chessell IP, Day NC. Changes in dorsal root ganglion CGRP expression in a chronic inflammatory model of the rat knee joint: differential modulation by rofecoxib and para- 
cetamol. Eur J Pain 2007; 11: 283-9.

36 Walsh DA, Bonnet CS, Turner EL, Wilson D, Situ M, McWilliams DF. Angiogenesis in the synovium and at the osteochondral junction in osteoarthritis. Osteoarthritis Cartilage 2007; 15: 743-51.

37 Suri S, Gill SE, Massena de Camin S, McWilliams DF, Wilson D, Walsh DA. Neurovascular invasion at the osteochondral junction and in osteophytes in osteoarthritis. Ann Rheum Dis 2007; 66: 1423-8.
38 Ethgen O, Bruyere O, Richy F, Dardennes C, Reginster JY. Health-related quality of life in total hip and total knee arthroplasty. A qualitative and systematic review of the literature. J Bone Joint Surg Am 2004; 86: 963-74.

39 Yoneda T, Hata K, Nakanishi M, Nagae M, Nagayama T, Wakabayashi H, et al. Involvement of acidic microenvironment in the pathophysiology of cancer-associated bone pain. Bone 2011; 48: 100-5. 